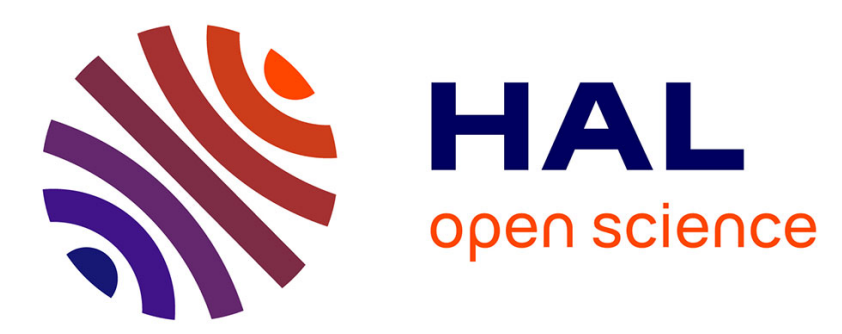

\title{
Preliminary Reflections on Affective Affordance in HCI: A Semiotic-Informed Perspective
}

Elaine Hayashi, Alessandro Arpetti, M. Baranauskas

\section{To cite this version:}

Elaine Hayashi, Alessandro Arpetti, M. Baranauskas. Preliminary Reflections on Affective Affordance in HCI: A Semiotic-Informed Perspective. 17th International Conference on Informatics and Semiotics in Organisations (ICISO), Aug 2016, Campinas, Brazil. pp.43-52, 10.1007/978-3-319-42102-5_5 . hal-01646562

\section{HAL Id: hal-01646562 \\ https://hal.inria.fr/hal-01646562}

Submitted on 23 Nov 2017

HAL is a multi-disciplinary open access archive for the deposit and dissemination of scientific research documents, whether they are published or not. The documents may come from teaching and research institutions in France or abroad, or from public or private research centers.
L'archive ouverte pluridisciplinaire HAL, est destinée au dépôt et à la diffusion de documents scientifiques de niveau recherche, publiés ou non, émanant des établissements d'enseignement et de recherche français ou étrangers, des laboratoires publics ou privés.

\section{(c)(1)}

Distributed under a Creative Commons Attribution| 4.0 International License 


\title{
Preliminary Reflections on Affective Affordance in HCI: A Semiotic-Informed Perspective
}

\author{
Elaine C. S. Hayashi, Alessandro Arpetti, and M. Cecília C. Baranauskas \\ Institute of Computing, University of Campinas (UNICAMP) \\ Campinas, SP, Brazil \\ \{hayashi, cecilia\}@ic.unicamp.br, arpetti@gmail.com
}

\begin{abstract}
In spite of some divergence on interpretations of the concept of "affordance", the term is fundamental in HCI and interaction design. More recently, the recognition of the affective quality of systems is becoming increasingly important as well. As an emerging subject, there is a lack of discussion on related concepts and, ultimately, on their practical implications. The objective of this paper is to bring into discussion the concept of affect under the perspective of affordance. From a socio-cultural view, this paper articulates affective affordances within the theoretical references of Organizational Semiotics and the Socially Aware Design; and concludes with prospections on potential applications for the design of technology.
\end{abstract}

Keywords: Affectability, Emotional design, Affordance, Affective affordances, Semiotic onion, Organizational Semiotics, Socially aware design, Humancomputer interaction

\section{Introduction}

Ubiquitous computing devices, wearable, tangible or natural user interface tools, social software: these are some of the ways in which Information and Communication Technology (ICT) has become a pervasive part of our personal and social lives. As an intrinsic part of our daily interactions and socially constructed reality, technology must be aligned with our intentions, values, beliefs and social practices. The design process focused only on the technical system itself is no longer enough [21]. This new reality demands new theories and methods in HCI [8, 17], which now have to consider and reflect other elements that used to be left aside, such as: social values, emotions, intentions of use, and the device's, the users' and the environment's conditions and characteristics $[14,17]$.

The concept of affordance, analyzed under an Organizational Semiotic (OS) perspective, has provided basis to inform the design of an inclusive social network [14]. Similarly, the present article builds upon the original definition of affordance from Gibson [6] to discuss design for Affectability [9]. At a higher level of abstraction, this article investigates the concept of affordance, proposing a discussion on Affective affordance framed by an artifact from OS: the Semiotic Onion [21, 13]. Although the term Affective affordance had been used before, e.g., in [23, 25, 12], the 
field of HCI could benefit from further discussion and informed formalization from a socio-technical perspective.

We revisit concepts of affordance and we use the perspective of the Semiotic Onion $[21,22,13]$ and the Socially Aware Design $[1,2]$ to articulate the concept of Affective affordance with possible design practices. This paper is organized as follows: Section 2 summarizes the concepts that form the theoretical bases of the analysis presented in Section 3, which articulates affect and affordances. Section 4 draws on Section 3 to discuss the application of these concepts in interaction design. Section 5 concludes this paper.

\section{$2 \quad$ Theoretical References}

Organizational Semiotics (OS) $[21,22,13]$ provides artifacts and methods to understand, model and design information systems. Information systems, from the OS perspective, are a result of social interactions. Starting from an informal level, a community (organization) is seen as a collection of members and the values, beliefs and habits of each member are relevant to form the group. In a formal level, the patterns of behavior from the informal level are modeled into norms that compose requirements in the direction of the technical layer, in which the system per se is derived. These informal, formal and technical layers form the Semiotic onion [21, 13]. The analogy with an onion illustrates how each level is embedded into the other to compose meaningful information systems, in which computer systems (or digital artifacts) are embedded in the formal and informal organization. As Stamper et al. [21] argue, usually traditional design methods tend to focus on the formal and technical levels - or layers - without meeting the requirements of the informal level of the organization.

The layers of the Semiotic Onion are rooted in the definition from Hall [7], who proposes that cultural conventions (the way humans operate) can be classified in informal, formal and technical levels.

The Socially Aware Design [1, 2], in its turn, was based on the Semiotic Onion to propose a design flow that starts in the society (with its needs and abilities), enters the onion, goes throughout the layers until reaching the technical one; and goes back to impact society, as technology changes the norms and informal practices of a social group.

The Semiotic Onion and the Socially Aware Design were the starting point for our research on affect in design processes, and they also form the basis for the discussion we present in Sections 3 and 4.

Affective computing [18] is a relatively new field that concerns the development of digital technology that can automatically recognize, interpret and respond (or adapt behavior) according to affective expressions of users. In order to grant computation systems with this ability to recognize affect, affective responses need to be categorized and treated as pieces of information. It is, therefore, assumed that emotions are internally created and then put out to the world, existing independently of context and interaction. Boehner et al. [3] call "Informational approaches" those approaches that are in line with Affective Computing, in which emotions are seen as fixed information. On a different direction, the authors call "Interactional approach" 
the point of view that considers affect as dynamic results of social interactions, loaded with cultural influences and rich in interpretation possibilities.

In line with interactional approaches, however not totally denying informational ones, the concept of Affectability [9] delineates an endeavor to understand the characteristics that have the capacity of evoking varying (which can depend on cultures or contexts) affective responses from users interacting with a digital artifact in a given context (or environment). The objective is to bring awareness to designers on the creation of products with improved affective quality.

Affective quality can be defined as the characteristics of an object (or place, events, systems) that can influence the affective state of a person [26], as he/she perceives those characteristics in the object. This perception can be based on how easy/hard to use, how pleasant/not pleasant, boring/exciting, etc., the object is.

Affordance is a concept created by Gibson [6], who defines it as the action possibilities that an animal perceives from an environment. With the word "affordance", Gibson wants to refer to both: the environment and the animal, in a way that one is complementary to the other. In Kaptelinin and Nardi's [11] interpretation of the Gibsonian theory of affordances, "animals directly perceive affordances as possibilities for action in the environment, which are determined, on the one hand, by the objective properties of the environment, and, on the other hand, by the action capabilities of the animal" [11, p. 968]. Moreover, as Gibson [6] explains, "The perceiving of an affordance is not a process of perceiving a value-free physical object to which meaning is somehow added in a way that no one has been able to agree upon; it is a process of perceiving a value-rich ecological object." [6, p. 60].

To Gibson, affordances exist independently of whether or not they are perceived or desired by the actor. The classic example is that of a chair. A chair affords sitting, even when the actor does not will to sit. Even when one cannot see the chair, it is still sit-able [24]. This constitutes a point of divergence between Gibson's and Norman's understanding of the concept. Norman [15] is more interested in processing mechanisms and how the mind processes the information perceived. There are authors who argue for separating affordances from perception, as it was originally proposed by Gibson [11,24]. The claim is that there is an advantage of considering the existence of affordance independently of it being perceived by the actor or not. This separation of affordances from perception helps to differentiate between two aspects of design elements: its affordance and its usability [11,24]. For Norman, "affordances can be not only real but also just perceived" [11, p. 969] that is only affordance itself, without being perceived, plays a relatively minor role as designers should be more concerned with what users can perceive [15].

The contrast between the various theoretical positions and the different interpretations of the concept of affordance can be analysed by going back to the work of Gibson in its roots, namely the theory of ecological perception. As we reported in reference to the concept of affordance by Gibson, the ecological term is understood in the sense of adaptation of an animal to its environment, or in the relations between individuals of a species, the organized activities of this species and the environment of these activities. In this sense, the environment is not the "external world", but is intended as 'ecological niche', that is, the behaviour of a species living under specific environmental conditions. 
This definition of affordance establishes a mutual connection between the animal and the environment, and the indivisibility between perception and action. Gibson himself [5] characterizes the perceptual system as a connection between the action and its perception. For the author, the perception is an emerging process of animalenvironment system. This implies that the perception does not reside in the brain, but is a process of extracting information from the environment, by means of exploration. In fact, if the primitive affordances linked to survival instincts are innate, all the others are the result of exploration of the environment through which the animal with its characteristics and capacity for action relates to an environment with specific characteristics. The information derived from this exploration is already meaningful information, since it is an element of the animal-environment system and is not compatible with the internalist interpretation proposed by cognitivism, according to which this information is a description of the environment that will be processed by the mind to give it a meaning.

Stoffregen [20] who provides a formal definition of affordances says that the emergent properties of the system are not present individually in the actors that are part of the system. For example, if we consider the climbability (affordance) of stairs, we should consider a system composed by a person (animal), the action of climbing (action) and the stairs (environment). The person and the stairs have individual properties (length of the leg, height of the step), but the system has a unique feature that is not present individually in any of the two actors, and is the scalability of the step, namely the relationship between the height of the step and the length of the person's leg.

The affordances therefore suggest possible behaviours that a system allows, but Stroffregen [20] states that it is the "psychological choice" of the actor that will bring the effective implementation of the action. In this choice, the emotions and the affective states can participate and produce a relationship with either a particular property of the environment to create an affordance or as part of a behaviour, by means of the consequences that an action produces in a person.

\section{Affective Affordance}

Extending the formalization of the concept of affordances proposed by Stroffregen [19], we can define an affective affordance by introducing an affective response of the user:

Let $\mathrm{W}_{p q}$ (e.g., a user-interaction-artifact system $)=\left(\mathrm{X}_{p}, \mathrm{Z}_{q}\right)$ be composed of different things $X$ (e.g., user) and Z (e.g., artifact).

Let ${ }_{p}$ be a property of $\mathrm{X}$ and ${ }_{q}$ be a property of $\mathrm{Z}$.

The relation between ${ }_{p}$ and ${ }_{q}, p / q$, defines a higher order property (i.e., a property of the user-artifact system), $h$.

Then $h$ is said to be an affective affordance of $\mathrm{W}_{p q}$ if and only if:

(i) $\mathrm{W}_{p q}=\left(\mathrm{X}_{p}, \mathrm{Z}_{q}\right)$ possesses $h$

(ii) Neither $Z$ nor $X$ possesses $h$

(iii) $h$ produces an affective response $\mathrm{A}(\mathrm{X})$. 
The main difference between a general affordance and an affective affordance lies in the fact that a general affordance is directly linked to a behavior, while an affective affordance produces an emotional state that can or cannot precede the eventual psychological choice of implementing a behavior.

Considering the environment as ecological niche and the user as participant of a group, we can argue that affective responses are influenced by culture and society and depend on varied factors. As Boehner et al. [3] argue, the sense people make out of feelings and other affective states are intertwined with the way such emotional episodes are experienced and to the way they are translated into words. These processes happen both in the individual and collectively. While emotions come from inside an individual (as a result of either internal or external stimuli), it is in the collectivity that the meanings and translations into words (or gestures - especially in the case of sign languages or other cultural gestures, images, sounds, etc.) are formed and shared.

Affect, emotions and the words that attempt to define them have a social and cultural nature. Boehner et al. [3] give as an example the concept of "song", from the Ifaluk Atoll. "Song" could be directly translated to "anger". However, while in a Western context, "anger" has a rather negative connotation, the Ifalukan "song" is considered a justifiable anger, as it is provoked by someone's lack of responsibility or failure to properly respond to social norms.

Similarly, affective responses also have cultural background. The Sensual Evaluation Instrument (SEI) [10], a self-assessment tool for affect, was created based on the premise that emotions can be explored from a non-verbal perspective as well as they can be evoked by different stimuli, like colors and shapes. The authors created and experimented objects with different shapes. In their experiments they observed that objects with sharp edges tended to be related to negative valence. On the other hand, objects with smooth surface and few protrusions were often associated to positive valence. The authors believe that SEI could be used across cultures and indeed one can easily recall personal experiences that validate those examples (that sharp edged objects are often related to negative affective responses; and smooth surfaces, to positive affective responses). While it is true and interesting for that selfassessment instrument, other objects with similar shapes can evoke opposite responses, which roots might be considered cultural or dependent on specific contexts. Fig. 1 illustrates this idea: it can be said that a baby hedgehog tends to have positive affective affordance, even though it has a prickly appearance. Other similar shaped objects would probably evoke not-touching. On the opposite way, maggots have soft looking surfaces but they tend to have negative affective affordance, usually evoking disgust or aversion. These responses are culturally learned. Babies inspire care; worms and larvae, disgust. Take other animals for examples. Guinea pigs are adorable pets in some countries. In other countries, they are a traditional dish, usually grilled as a whole. Cows are sacred in some cultures, tortured to death for meat in others. These contrasting ways to treat animals are socially accepted in their own cultures and cause people with different background to present different affective responses. 

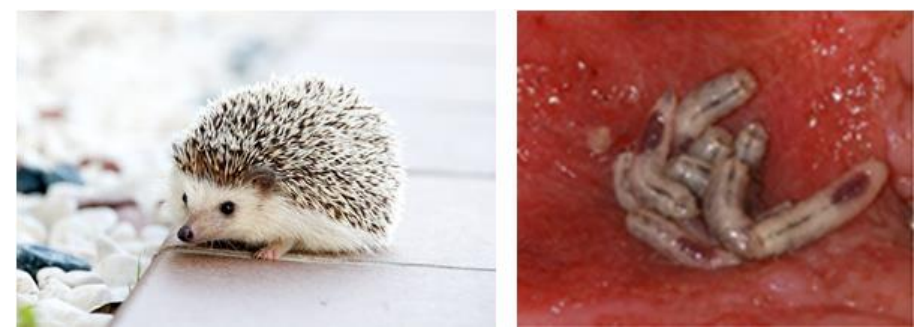

Fig. 1. In spite of its prickly appearance, a hedgehog baby ${ }^{1}$ might have positive affective affordance, whereas maggots ${ }^{2}$, with its soft appearance, would evoke negative affective affordance - contrary to the notions of what spiky and smooth objects usually evoke.

[Sources: ${ }^{1}$ Hedgehog baby https://pixabay.com/en/hedgehog-animal-baby-cutesmall-468228/;

${ }^{2}$ Maggots: https://commons.wikimedia.org/wiki/File:Maggots.jpg]

From an ecological perspective, this scenario becomes even more complex since user-system interactions are contaminated not only by socio-cultural elements but also by the individual's life experience. In the light of the formal definition of affective affordance in which $\mathrm{W}_{p q}=\left(\mathrm{X}_{p}, \mathrm{Z}_{q}\right)$. It is arguable that the image $\mathrm{Z}$ (the artifact, in this case a baby hedgehog (cf. Fig. 1)) has some characteristics ${ }_{\mathrm{q}}$ (colors, size, lights, position, background, etc.). The user $\mathrm{X}$ has some characteristics $\mathrm{p}$ (physical characteristics, social values, beliefs, affective state, etc.). The system $\mathrm{W}_{\mathrm{pq}}$ (userrelationship-image) affords an affective response $\mathrm{A}(\mathrm{X})$ which may or may not be expressed in behavior. This affective response is strongly linked to the system to all the meanings contained in it. For example, the image can afford a sentiment of sweetness in a young parent that can smile and eventually share the image in social media; or it can afford a feeling of disgust in a user who has just had a bad experience with a small rodent and, viewing the image, grimaces and shuts down the computer.

Affective responses are therefore the result of combinations of numerous elements in a complex system. To overcome the fragmentation of these responses and provide useful guidelines for interaction designers it is necessary to analyze, in a systemic way, the influences of the different actors in the formation of affective affordances.

\section{Affective Affordance and the Design of Digital Artifacts}

Affective responses could be analyzed within the Semiotic Onion and the flow of the Socially Aware Design. Take the example of Internet memes, i.e., media that are spread in the Internet, usually for fun. The "success kid" (Fig. 2a) is an example of Internet meme. It started as a common social action of taking pictures and a spontaneous affective expression that starts at the outmost layer of the semiotic onion: the informal layer. In order to reach a digital format and gain space online (technical layer), the picture was once treated and meanings were formalized with texts (formal layer). Going back the path towards the society, the online use of the Success kid meme gained a specific format (formal layers) as shown in Fig. 2b. As it became viral and worldwide known (informal layer), it even became theme for advertisement, in a billboard add. Also the White House in the United Stated used the meme, in a Tweet 
in their campaign to urge Congress to pass the immigration reform (Fig. 2c) representing the impact in society.
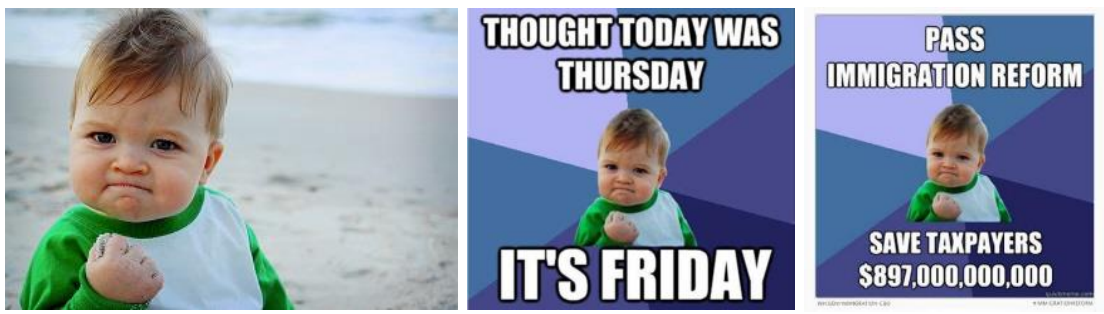

Fig. 2. From left to right: (a) original picture taken by the child's mother;

(b) internet meme example; (c) meme used by the USA White House in a Tweet

[Sources: (a) and (b) http://globalnews.ca/news/1939419/success-kid-needs-yourhelp-getting-his-dad-a-new-kidney/;

(c) http://www.nydailynews.com/news/politics/success-kid-meme-passimmigration-reform-article-1.1376952, Accessed: April 2016]

While it is hard to determine whether a product will be successfully accepted by users, one can investigate elements that can be of influence in the process. Tang et al [23] have shown the relevance of consumers' perceived affordances (namely physical, cognitive, affective and control affordances) in the success of some specific ads in China. We argue that affective affordances have the potential to influence the design of ICT in general. The design for Affectability [9] is the design that is concerned with affective affordances throughout the entire design process. We had presented the concept of Affectibility and the Framework for Affectibility based on the same theoretical roots explained in Section 2. Next we present the creation and development of an application, which illustrates the design towards improved Affectibility - that is, the design that is concerned with the possible affective affordances that could be collectively constructed and perceived by users.

\subsection{Designing AmigoDaHora}

We had been immersed in an underprivileged community of a public school in Brazil, taking active part in the school's daily activities as participant observers. From this endeavor we identified many characteristics of that group of children; one of them was the very noticeable tendency to negative thoughts and behavior. Not only children but also teachers and other employees from the school were often prone to see defects and points of complaint in almost any subject. Seeing positive aspects was difficult for them. Following the flow of the Socially Aware Design [1], this predominance of rather negative affective affordances in schools interactions can be seen as a characteristic from the school as a group (society). From this need (of practicing more positive views and attitudes) we proposed the creation of an application based on a social activity (informal layer) known as "Secret Santa" ("Amigo secreto" or "Amigo oculto" in Brazil). In this practice, each participant raffles the name of another and he/she has to buy the other a present. In our proposed 
version, each participant should gift the other a compliment, creating a gift representing that compliment.

Children played the role of our design partners in the development of the application. In participatory practices they helped us to better understand and formalize the rules of this new activity of gifting compliments (formal layer). By enacting the activity we tried to understand what elements the interface should represent as to provide the necessary characteristics to help achieve the desired affective responses. Children informed us not only about what they needed the application to have in order to create the gifts as they wanted, but they also named the application: AmigoDaHora - slang meaning something similar to "cool friend" (cf. Fig. 3a).

The technical layer is the application itself. We developed a mobile application that made use of RFID cards for tangible interaction. Only one mobile was needed and it was based on Android platform. Each child would have their own card. We asked children to personalize the cards by hand drawing something to represent them on the card. The application would read the card and identify each child as participant of AmigoDaHora. After registering all participants for a round (cf. Fig. 3b), the application would secretly assign the participants to each other. Each child would then take turns to privately interact with the application in order to find out who his/her friend was and create a gift/compliment for that friend (cf. Fig. 3c). The application let children speak the compliment out loud and it transcribed what they said. Children could also take pictures and/or make drawings as to compose the gift/compliment. After all children had created their gifts, they could take turns again to use the application and receive their gifts/compliments.

Going back from the technical layer (the development and evaluation of AmigoDaHora application) to society, we observed the use made of the application. Right on the first round playing with AmigoDaHora, children organized themselves and set rules to determine how they would take turns to interact with the application (formal layer). Sharing the single mobile device and making use of the application in the group (informal layer) should potentially direct children to the desired outcome of practicing seeing positive aspects in others and develop positive attitudes (impact back on society).

The factors (e.g., aesthetical, motivational, etc.) that might contribute to the establishment of positive affective affordances were listed in the form of design principles for Affectability (PAff), which were followed in the creation of AmigoDaHora. Among the six principles presented [9], for example, the PAff 1, Free interpretation and communication of affect, suggests to i) make available features to allow communication and expression; ii) avoid predetermining meanings (of signs, words, images, etc.) and let affect be freely expressed and interpreted; and iii) avoid automatic identification of affect. One affective affordance of the AmigoDaHora mobile application $(\mathrm{A}(\mathrm{X}))$ could be: affective expression by drawing smile faces with the finger. This possibility exists from $X_{p 1}=$ children fingers, $Z_{q 1}=$ touch screen, and $\mathrm{pl} / \mathrm{q} 1=$ children application on a mobile device for the creation and exchange of compliments. 

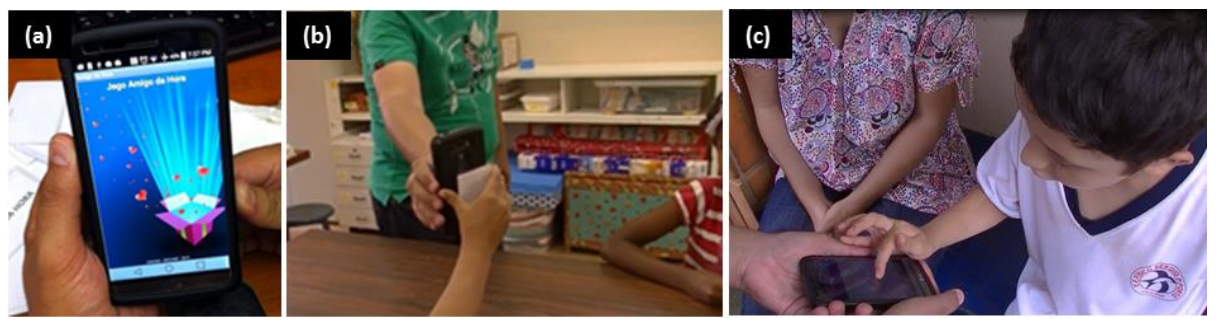

Fig. 3. (a) Main screen of AmigoDaHora (b) enrolling a child in the game (c) child drawing a smile face to illustrate his gift/compliment

\section{Conclusion}

Digital artifacts have to make sense to users. The Socially Aware Design argues for design processes that start in the society, with the understanding of the main stakeholders and the comprehension of the target users' main abilities and needs. In OS it is by understanding the informal practices of a social group that practices can be translated into the designed artifact.

Being affective responses a result of cultural and social interactions, in order to aim for improved Affectibility in the resulting solutions, designers could start their analysis from the society as well, following the path of the design throughout the Semiotic Onion. This approach can not only contribute to the creation of more meaningful systems, but also help designers having in mind the possible affective outcomes that the designed system might have when it, in its turn, influence back social practices. This article discussed the concept of Affective affordances, presenting a definition from an ecological perspective in which the environment and users are mutually connected, although perception and action are not always necessarily directly linked. Future work could explore the norms that help inform the design for improved Affectibility.

Acknowledgments. We would like to thank our colleagues from InterHAD for insightful discussions; CAPES and CNPq (Process \# 308618/2014-9) for financial support.

\section{References}

1. Baranauskas, M.C.C.: Social Awareness in HCI. Interactions 21(4), 66-69 (2014)

2. Baranauskas, M.C.C.: Socially Aware Computing. In: The $6^{\text {th }}$ International Conference on Engineering and Computer Education, Buenos Aires, pp. 1-5 (2009)

3. Boehner, K., DePaula, R., Dourish, P., Sengers, P.: How Emotion is Made and Measured. International Journal of Human-Computer Studies, 65(4), 275-291 (2007)

4. Bødker, S.: Third-Wave HCI, 10 Years Later-Participation and Sharing. Interactions 22(5), 24-31 (2015)

5. Gibson, J.J.: The Senses Considered as Perceptual Systems. Boston, Houghton Mifflin (1966)

6. Gibson, J.J.: The Theory of Affordances. The People, Place, and Space Reader 56 (1979) 
7. Hall, E.: The silent language. New York: Doubleday (1959)

8. Harrison, S., Tatar D., Sengers, P.: The three paradigms of HCI. Proc. of ACM AltCHI'07, pp. 1-21 (2007)

9. Hayashi, E.C.S., Baranasukas, M.C.C.: 'Affectability' and Design Workshops: taking actions towards more sensible design. In: The $12^{\text {th }}$ Brazilian Symposium on Human Factors in Computing Systems, Brazilian Computer Society, pp. 3-12 (2013)

10. Isbister, K., Höök, K., Sharp, M., Laaksolahti, J.: The sensual evaluation instrument: developing an affective evaluation tool. In: The SIGCHI Conference on Human Factors in Computing Systems, ACM, pp. 1163-1172 (2006)

11. Kaptelinin, V., Nardi, B.: Affordances in HCI: toward a Mediated Action Perspective. In: The SIGCHI Conference on Human Factors in Computing Systems, ACM, pp. 967-976 (2012)

12. Krampen, M.: Semiotics in Architecture and Industrial/Product Design. Design Issues 5(2), 124-140 (1989)

13. Liu, K.: Semiotics in Information Systems and Engineering. Cambridge University Press, Cambridge (2000)

14. Neris, V.P.A, Baranauskas, M.C.C.: User Interface Design Informed by Affordances and Norms Concepts. In: The $12^{\text {th }}$ IFIP WG 8.1 International Conference on Informatics and Semiotics in Organizations, ICISO, pp. 133-140 (2010)

15. Norman, D.A.: Affordance, Conventions, and Design. Interactions 6(3), 38-43 (1999)

16.Norman, D.: Emotional Design: Why We Love (or Hate) Everyday Things. Basic Books, New York, (2004)

17. Pereira, R., Baranauskas, M.C.C., Liu, K.: On the Relationships between Norms, Values and Culture: Preliminary Thoughts in HCI. Information and Knowledge Management in Complex Systems, Springer International Publishing, pp. 30-40 (2015)

18.Picard, R.: Affective computing. Cambridge, MA: MIT Press (1995)

19. Stoffregen, T.A.: Affordances as Properties of the Animal-Environment System. Ecological Psychology 15, 115-134 (2003)

20. Stoffregen, T.A.: Breadth and limits of the affordance concept. Ecological Psychology 16, 79-85 (2004)

21.Stamper, R.: A Semiotic Theory of Information and Information Systems. Invited paper on Information by the ICL/University of Newcastle (1993)

22. Stamper, R., Liu, K., Hafkamp, M., Ades, Y.: Understanding the Roles of Signs and Norms in Organisations: a Semiotic Approach to Information Systems Design. Journal of Behavior and Information Technology 19 (1), 15-27 (2000)

23.Tang, J., Zhao, X., Zhang, P.: Perceived affordances of web advertisements: Implications for information artifacts design. Proc. of the Fifth China Summer Workshop on Information Management, pp. 25-26 (2011)

24.Torenvliet, G.: We can't afford it!: the Devaluation of a Usability Term. Interactions 10(4), $12-17$ (2003)

25. Vugt, H.C., Hoorn, J.F., Konjin, E.A., Dimitriadou, A.B.: Affective affordances: Improving interface character engagement through interaction. Journal of Human-Computer Studies 64, 874-888 (2006)

26.Zhang, P., Li, N.: The Importance of Affective Quality. Communications of the ACM. September 48(9), 105-108 (2005) 